\title{
DISTRIBUIÇÃO ESPACIAL E TAMANHO DE AMOSTRA PARA O ÁCARO-DO- BRONZEADO DA ERVA-MATE ${ }^{1}$
}

\author{
Alessandro Dal’ Col Lúcio², João Vieira Neto ${ }^{3}$, Luis Antônio Chiaradia ${ }^{4}$ e Lindolfo Storck ${ }^{2}$
}

\begin{abstract}
RESUMO - O ácaro Dichopelmus notus Keifer (Acari, Eriophyidae) provoca o bronzeamento e queda de folhas da erva-mate, reduzindo a produção e a qualidade de seus produtos. O acompanhamento dos níveis populacionais dessa espécie é importante para aprimorar seu manejo em plantios comerciais. Este trabalho teve por objetivos identificar a distribuição espacial e determinar o número de plantas e de folhas por planta que devem ser inspecionadas em cultivos comerciais de erva-mate em programas de monitoramento do ácaro-do-bronzeado. O estudo foi conduzido no Município de Chapecó, Santa Catarina, no período de janeiro de 2004 a janeiro de 2005. As avaliações foram realizadas quinzenalmente em um erval de 10 anos, dividido em três talhões com cerca de $2.500 \mathrm{~m}^{2}$ cada, em que foram selecionadas 10 plantas ao acaso e em cada planta foi observado o número de ácaros em 18 folhas maduras. As inspeções foram executadas diretamente nos ervais com lentes com aumento de 10 vezes e $1 \mathrm{~cm}^{2}$ de campo fixo. Constatou-se a distribuição espacial agregada para o ácaro, bem como a necessidade de inspecionar três folhas por planta, em 29 delas ao acaso e por hectare, de fevereiro a abril, para estimar a população com nível de precisão de $15 \%$.
\end{abstract}

Palavras-chave: Amostragem, Ilex paraguariensis e Dichopelmus notus.

\section{SPATIAL DISTRIBUTION AND SAMPLE SIZE FOR THE TAN-MITE IN MATE-TEA}

\begin{abstract}
The mite Dichopelmus notus Keifer (Acari, Eriophyidae) causes tan and fall of leaves in mate trees, reducing the production and quality of their products. Monitoring the population levels of this type of mite is important to improve its management in commercial plantations. The objective of this work was to identify the spatial distribution and to determine the number of plants and leaf per plant that must be inspected in commercial cultivation of mate-tea in tan mite monitoring programs. The study was conducted in Chapecó, Santa Catarina, from January 2004 to January 2005. The evaluations were performed fortnightly in a 10-year old orchard, divided into three plots of approximately $2.500 \mathrm{~m}^{2}$. In each plot, ten plants were randomly selected and in each, the number of mites was observed in 18 mature leaves. The evaluations were carried out directly in the orchard, using 10-fold increase lenses and $1 \mathrm{~cm}^{2}$ of fixed field. Spatial distribution aggregate for the mite was confirmed, as well as the need to inspect three leaves per plant, 29 of them at random per hectare, from February to April in order to estimate the population at a level of accuracy of $15 \%$.
\end{abstract}

Keywords: Sampling, Ilex paraguariensis and Dichopelmus notus.

\section{INTRODUÇÃO}

A erva-mate, Ilex paraguariensis St. Hil. (Aquifoliaceae), ocorre nas regiões temperadas e subtropicais da América do Sul, predominante nos Estados de Santa Catarina, Paraná e Rio Grande do Sul, Brasil. Essa planta foi durante muitos anos extraída de ervais nativos e recentemente passou a ser cultivada em monocultura, o que pode favorecer as pragas (PENTEADO, 1995). O ácaro-do-bronzeado Dichopelmus notus Keifer (Acari, Eriophyidae), praga que apresenta bastante afinidade com a erva-mate, provoca o bronzeamento e queda prematura de suas folhas e a morte de ponteiros com prejuízos frequentes, dependendo da intensidade de infestação (SANTANA et al., 1997).

\footnotetext{
${ }^{1}$ Recebido em 30.03.2007 e aceito para publicação em 26.01.2009.

${ }^{2}$ Departamento de Fitotecnia da Universidade Federal de Santa Maria (UFSM). E-mail: <adlucio@ smail.ufsm.br>.

${ }^{3}$ Programa de Pós-Graduação em Agronomia da UFSM. E-mail: <joaovieira@ epamig.br>.

${ }^{4}$ Empresa de Pesquisa Agropecuária e Extensão Rural de Santa Catarina S. A. (EPAGRI). E-mail: <chiaradi@epagri.sct-sc.br>.
} 
O acompanhamento dos níveis de infestação de $D$. notus é importante para aprimorar seu manejo.

A avaliação da densidade populacional requer a contagem da espécie por unidade amostral (numérica) ou, de maneira alternativa, verificar se está presente na unidade amostral (binomial) (WILSON et al., 1983; WILSON et al., 1984; PEREIRA et al., 2004). Nesse contexto, a amostragem exerce papel importante no monitoramento de pragas em lavouras comerciais (WILSON; ROOM, 1983). A escolha do método de amostragem depende do custo operacional e da precisão que ele oferece ou da combinação desses fatores (SILVEIRA; NETO et al., 1976).

A distribuição espacial dos indivíduos em seu habitat afeta planos de amostragem, exigindo métodos diferenciados de acordo com as particularidades de cada espécie (RUESINK; KOGAN, 1980). Vários índices têm sido utilizados para determinar a distribuição espacial de insetos e ácaros. O coeficiente "b", obtido por regressão através da lei da potência de Taylor (TAYLOR, 1961), tem sido frequentemente utilizado. Esse método estabelece a relação entre a variância e a média de populações expressa por $S^{2}=a^{b}$, em que $S^{2}$ é a estimativa da variância populacional, $m$ é a estimativa da média populacional e "a" e "b" são os coeficientes do modelo. O coeficiente $\beta$ (LLOYD, 1967), estimado por regressão do índice de agrupamento $\mathrm{m} *$, pela média populacional, com o modelo $\mathrm{m}^{*}=\alpha+\beta \mathrm{m}$ é também utilizado, em que $\mathrm{m}^{*}$ corresponde a $\mathrm{m}^{*}=\mathrm{m}+\left(\mathrm{S}^{2} / \mathrm{m}\right)-1$, mé a estimativa da média populacional e $\alpha$ e $\beta$ são os coeficientes do modelo. Valores resultantes a 1 para os coeficientes "b" e $\beta$ indicam distribuição ao acaso; para "b" e $\beta$ $>1$, distribuição agregada; e para "b" e $\beta<1$, distribuição uniforme (TAYLOR, 1961; IWAO, 1972). Os dados referentes à contagem de pragas ou de seus danos com distribuição uniforme podem ser estudados pela distribuição de probabilidade discreta binomial, que se caracteriza por apresentar variância menor que a média. Para disposição aleatória, pode-se utilizar a distribuição discreta de Poisson, cujos dados apresentam variância e média semelhantes, e, no caso de disposição agregada, utilizam-se distribuições discretas de contagem (variância maior que a média), sendo mais comum a distribuição binomial negativa (BARBOSA, 2001).

Vários métodos foram desenvolvidos para o dimensionamento do tamanho das amostras, sendo os mais utilizados o método da máxima curvatura do

R. Árvore, Viçosa-MG, v.33, n.1, p.143-150, 2009 coeficiente de variação (REYNOLDS et al., 1934); a equação de Karadinos (1976), modificada por Ruesink e Kogan (1980) e Wilson e Roon (1983) e o método proposto por Cochran (1977). Assim, este trabalho teve como objetivos determinar a distribuição espacial e o tamanho da amostra para estimar a densidade populacional do ácaro-do-bronzeado em cultivos comerciais de erva-mate, nas condições do Município de Chapecó, SC.

\section{MATERIAL E MÉTODOS}

O estudo foi conduzido em um erval de 10 anos da empresa Ouro Verde, no distrito de Marechal Bormann, Município de Chapecó, Santa Catarina, com árvores em espaçamento 2,5 x 4,0 m e altura de poda de 1,5 $\mathrm{m}$. Quinzenalmente, no período de janeiro de 2004 a janeiro de 2005, em talhões de aproximadamente 2.500 $\mathrm{m}^{2}$ distantes entre si cerca de $100 \mathrm{~m}$, foram selecionadas 30 plantas ao acaso (10 em cada talhão). A infestação da praga foi avaliada em 18 folhas maduras de 10 plantas em cada talhão, sendo seis no terço superior, seis no médio e seis no inferior, três em cada localização da copa, externa e interna. As inspeções foram executadas, diretamente, nos ervais, com lentes com aumento de 10 vezes e $1 \mathrm{~cm}^{2}$ de campo fixo. As estimativas da média $(\mathrm{m})$, variância $\left(\mathrm{S}^{2}\right)$ e o índice de agrupamento $\mathrm{m} *\left(\mathrm{~m}^{*}\right.$ $\left.=\mathrm{m}+\left(\mathrm{S}^{2} / \mathrm{m}\right)-1\right)$ foram calculadas para o número de ácaros por folha (540 folhas) e planta (30 plantas), em cada data de amostragem. Em seguida, foram ajustadas ao modelo de Taylor (1961) e ao modelo de Lloyd (1967), para obtenção das estimativas dos parâmetros de regressão.

O tamanho da amostra para número de folhas e para o número de plantas foi determinado com a equação modificada de Karadinos (1976), expressas por: $\mathrm{n}=(\mathrm{t} / \mathrm{D})^{2} \cdot \mathrm{am}^{\mathrm{b}-2}$, em que $\mathrm{n}$ é o tamanho da amostra; $\mathrm{t}_{\alpha / 2}$ um valor da tabela $\mathrm{t}$, de Student, para um nível $\alpha$ de significância, adotado 5\%; D (semiamplitude do intervalo de confiança da média) igual a 5, 10, 15, 20 e $30 \%$ da média estimada nos levantamentos amostrais; "a" e "b" são os coeficientes de Taylor; e m, a média do número de ácaros por unidade amostral. Utilizouse também a expressão proposta por Cochran (1977): $\mathrm{n}=\mathrm{Nt}^{2}(\mathrm{CV} \%)^{2} / \mathrm{N}(\mathrm{D})^{2}+\mathrm{t}^{2}(\mathrm{CV} \%)^{2}$, em que n é o tamanho da amostra; N é o número total de plantas da população (30 plantas); $\mathrm{t}_{\alpha / 2}$ é um valor da tabela $\mathrm{t}$, de Student, para um nível $\alpha$ de significância, adotado 5\%; CV\% é o coeficiente de variação; e D (semiamplitude do 
intervalo de confiança da média) igual a 5, 10, 15, 20 e $30 \%$ da média do número de ácaros por unidade amostral.

Para testar a homogeneidade das variâncias entre as unidades amostrais (folha e planta) dentro de cada data de avaliação, foi utilizado o teste de Bartlett (STEEL et al., 1997). Quando o teste de Bartlett acusou homogeneidade de variâncias entre as unidades amostrais, o coeficiente de variação foi estimado com a média das variâncias pelo grau de liberdade; caso contrário, utilizou-se a variância máxima.

\section{RESULTADOS E DISCUSSÃO}

As médias do número de ácaros estimadas em cada data de amostragem em área total, para folha e planta, mostraram maior densidade populacional do ácaro $D$. notus entre o final de fevereiro e meados de maio, com pico populacional no final de março e início do mês de abril, quando foi constatada, em média, 10,25 ácaros por $\mathrm{cm}^{2} \mathrm{de}$ folha (Figura $1 \mathrm{AB}$ ). Essas ocorrências podem estar associadas às temperaturas e precipitações mais moderadas do outono.

As variâncias do número de ácaros tendem a acompanhar o comportamento das médias, com maiores valores no período de maior densidade populacional do ácaro e vice-versa (Figuras 2AB). Essa relação é comum, sendo utilizada para definir metodologias para detectar o índice de agregação de insetos e ácaros - lei da potência de Taylor e o modelo de regressão de Lloyd (RUESINK e KOGAN, 1980; RIBEIRO, 1990; BEARZOTI e AQUINO, 1994; VIEIRA NETO e CHIARADIA, 1999; FARIAS et al., 2001; MARUYAMA et al., 2002; SILVA et al., 2003; ONZO et al., 2005). Isso reforça a consistência dessas metodologias para estudar o tipo de distribuição espacial do ácaro D. notus em pomares de erva-mate.
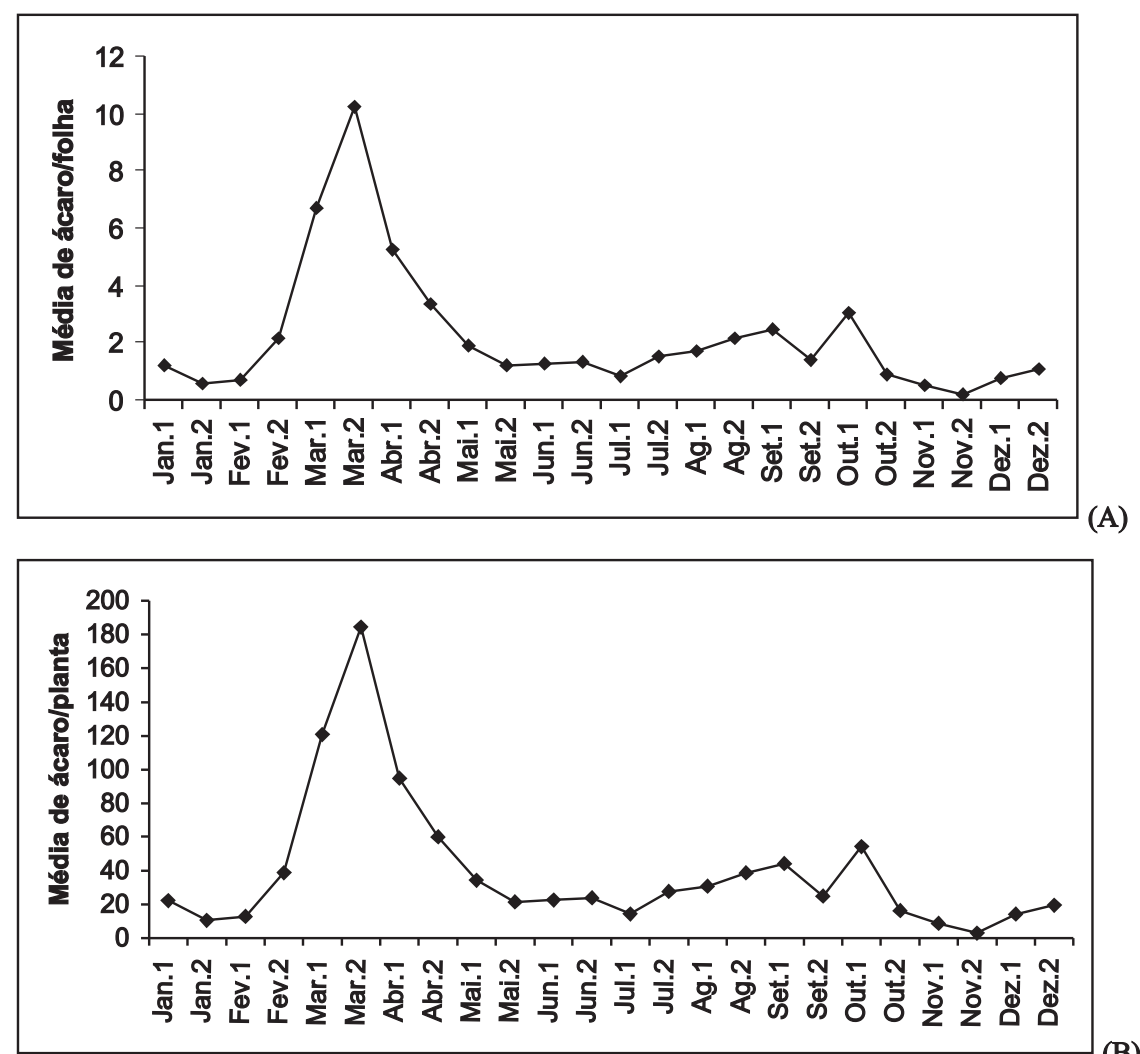

Figura 1 - Número de ácaros Dichopelmus notus por folha (A) e por planta (B) na área total em plantios comerciais de erva-mate. Chapecó, SC, 2006.

Figure 1 -Number of Dichopelmus notus mites per leaf $(A)$ and plant $(B)$ in the total area in commercial plantations of mate-tea. Chapecó, SC, 2006. 


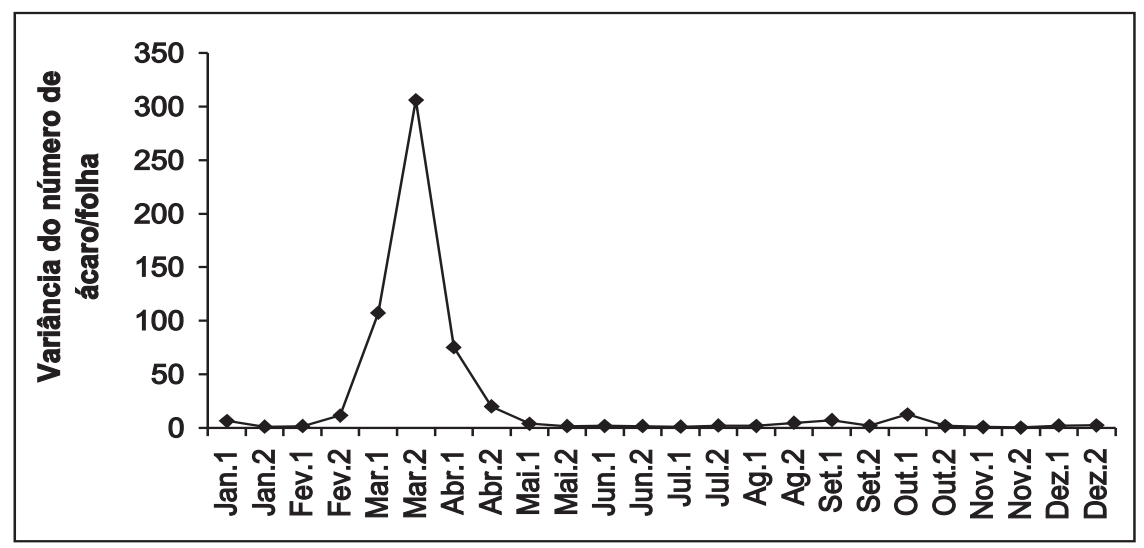

(A)

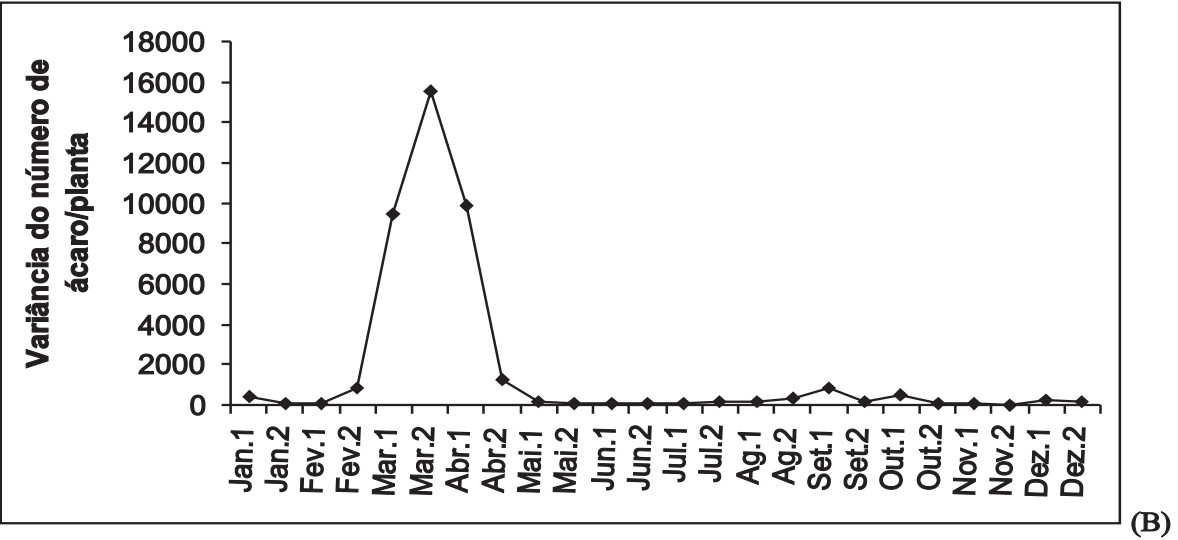

Figura 2 - Variância por folha (A) e por planta (B) do número de ácaros Dichopelmus notus na área total em plantios comerciais de erva-mate. Chapecó, SC, 2006.

Figure 2 - Variance per leaf $(A)$ and per plant $(B)$ of the number of Dichopelmus notus mites in the total area in commercial plantations of mate-tea. Chapecó, SC, 2006.

Os dois modelos de Lloyd e Taylor apresentaram bons ajustes, mas os coeficientes de determinação $\left(\mathrm{R}^{2}\right)$ indicam que o modelo de regressão do primeiro apresentou melhor desempenho do que o segundo, para folha e planta de erva-mate, em todos os locais (Tabela 1). As situações analisadas tiveram " $b$ " e $\beta$ maiores que 1 , indicando que a distribuição espacial do ácaro no erval é agregada, tanto para folha quanto para a planta. Isso é semelhante ao padrão de distribuição espacial do ácaro Panonychus ulmi (Koch) (Acari: Tetranychidae) em pomar de macieiras (RIBEIRO, 1990) e para o ácaro Tetranychus urticae (Acari: Tetranychidae) em morangos, em La Plata (Buenos Aires) (GRECO et al., 1999). Os ácaros Mononychellus tanajoa (Bondar) (Acari: Tetranychidae) e Typhlodromalus aripo (DeLeon) (Acari: Phytoseiidae) também apresentaram padrão espacial agregado na cultura da mandioca, em Benin, oeste da
África (ONZO et al., 2005). A baixa mobilidade da maioria dos ácaros fitófagos pode justificar esse padrão de distribuição. Mas a capacidade de dispersão dessas espécies não deve ser negligenciada, por serem capazes de estabelecer focos de infestação em que estejam presentes.

A disposição espacial dos indivíduos no habitat afeta a amostragem, tornando necessário o uso de diferentes planos para estratégias de manejo, incluindo escolha da metodologia para tomada de decisão em amostragem sequencial. O padrão agrupado, apresentado por esse ácaro, torna necessário o uso de distribuições discretas de contágio. No entanto, na metodologia baseada em intervalos de confiança para média e proporção deve-se optar por modelos com algum índice de agregação da praga, especialmente quando forem do tipo uniforme ou agregado, com índices diferentes da unidade. 
Tabela 1 - Coeficientes lineares, de regressão e de determinação dos ajustes à regressão $\mathrm{m} * \mathrm{~m}$ e à lei da potência de Taylor, a partir do número de ácaros Dichopelmus notus observados por folha e por planta de erva-mate. Chapecó, SC, 2006

Table 1 - Linear, regression and determination coefficients of the adjustments to regression $m * / m$ and to the Taylor power law, from the number of number of Dichopelmus notus mites per leaf and plant of mate-tea. Chapecó, SC, 2006

\begin{tabular}{|c|c|c|c|c|c|c|}
\hline \multirow[t]{2}{*}{ Local de amostragem } & \multicolumn{3}{|c|}{$\operatorname{Ln} S^{2}=\ln a+b \cdot \ln m$} & \multicolumn{3}{|c|}{$\mathrm{m}^{*}=\alpha+\beta \mathrm{m}$} \\
\hline & Ln a & $\mathrm{b}$ & $\mathrm{R}^{2}$ & $\alpha$ & $\beta$ & $\mathrm{R}^{2}$ \\
\hline \multicolumn{7}{|c|}{ Estimativas por folha } \\
\hline Talhão 1 & $\begin{array}{c}0,6858 \text { * } \\
(0,1525)^{\mathrm{I}}\end{array}$ & $\begin{array}{l}1,5959^{*} \\
(0,1510)\end{array}$ & 0,8353 & $\begin{array}{c}-1,7185^{*} \\
(0,5241)\end{array}$ & $\begin{array}{l}3,1965^{*} \\
(0,1748)\end{array}$ & 0,9382 \\
\hline Talhão 2 & $\begin{array}{l}0,5521 \\
(0,1288)\end{array}$ & $\begin{array}{c}1,6746^{*} \\
(0,1316)\end{array}$ & $\begin{array}{c}0,8803 \\
(0,6049)\end{array}$ & $\begin{array}{l}-2,2720^{*} \\
(0,1646)\end{array}$ & $3,2482 *$ & 0,9465 \\
\hline Talhão 3 & $\begin{array}{l}0,6802^{*} \\
(0,1559)\end{array}$ & $\begin{array}{l}1,8221^{*} \\
(0,1618)\end{array}$ & $\begin{array}{c}0,8520 \\
(0,9205)\end{array}$ & $\begin{array}{c}-3,5180 * \\
(0,3171)\end{array}$ & $4,5985^{*}$ & 0,9052 \\
\hline Área total & $\begin{array}{l}0,7017^{*} \\
(0,1384)\end{array}$ & $\begin{array}{l}1,7579 * \\
(0,1444)\end{array}$ & $\begin{array}{c}0,8706 \\
(0,5323) \\
\end{array}$ & $\begin{array}{c}-2,4543^{*} \\
(0,1702)\end{array}$ & $3,7702 *$ & 0,9572 \\
\hline \multicolumn{7}{|c|}{ Estimativas por planta } \\
\hline Talhão 1 & $\begin{array}{c}-0,5502^{\mathrm{NS}} \\
(0,5257)^{\mathrm{I}}\end{array}$ & $\begin{array}{l}1,8535^{*} \\
(0,1585)\end{array}$ & $\begin{array}{c}0,8614 \\
(4,7830)\end{array}$ & $\begin{array}{c}-9,6523^{\mathrm{NS}} \\
(0,0872)\end{array}$ & $1,7463^{*}$ & 0,9479 \\
\hline Talhão 2 & $\begin{array}{c}-0,4166 \text { Ns } \\
(0,7463)\end{array}$ & $\begin{array}{l}1,7776^{*} \\
(0,2195)\end{array}$ & $\begin{array}{c}0,7486 \\
(2,8532)\end{array}$ & $\begin{array}{c}-6,8277^{*} \\
(0,0503)\end{array}$ & $1,5746^{*}$ & 0,9779 \\
\hline Talhão 3 & $\begin{array}{c}-0,2083 \text { Ns } \\
(0,5577)\end{array}$ & $\begin{array}{l}1,7301 * \\
(0,1622)\end{array}$ & $\begin{array}{c}0,8379 \\
(5,4698)\end{array}$ & $\begin{array}{c}-3,1527 \text { NS } \\
(0,0917)\end{array}$ & $1,4895^{*}$ & 0,9229 \\
\hline Área total & $\begin{array}{c}-0,2894{ }^{\mathrm{NS}} \\
(0,5758)\end{array}$ & $\begin{array}{l}1,8047^{*} \\
(0,1694)\end{array}$ & $\begin{array}{c}0,8375 \\
(4,1288)\end{array}$ & $\begin{array}{c}-4,3013 \text { Ns } \\
(0,0733)\end{array}$ & $1,5775^{*}$ & 0,9545 \\
\hline
\end{tabular}

I - Valor entre parênteses refere-se ao erro-padrão da estimativa. NS - não-significativo.

* - Significativo a $5 \%$ de probabilidade de erro, pelo teste " $t$ ".

Outra implicação prática da dispersão de pragas está relacionada diretamente aos trabalhos de inspeção dos níveis populacionais no campo. Ao realizar a inspeção de espécie que apresenta padrão agregado de distribuição, é necessário, primeiramente, identificar o foco de ocorrência da praga na lavoura, uma vez que ela ocorre em reboleiras. Caso isso não seja observado, corre-se o risco de amostrar talhões onde a praga não esteja presente ou tenha baixa infestação. A simples recomendação de caminhamento em ziguezague, a partir de um ponto qualquer da lavoura, não seria recomendável nessa situação. Esse procedimento deverá ser adotado em casos em que ocorrem manifestações de padrão uniforme ou aleatório.

As estimativas do tamanho da amostra para folha e planta estão apresentadas nas Tabelas 2 e 3, com cálculos efetuados na área total, pois o padrão da distribuição do acarino em função do tamanho da área de amostragem não foi alterado.

O modelo de regressão de Taylor apresentou bons ajustes para a folha e planta, em todos os locais de amostragem com coeficiente de regressão significativo a 5\% de probabilidade pelo teste " $t$ " e com coeficiente de determinação $\left(R^{2}\right)$, em sua maioria, superior a 0,80 . No entanto, ao considerar a planta como unidade de amostragem os valores do coeficiente "a" não diferiram de zero pelo teste " $t$ " (Tabela 1). Por isso, os valores gerados pela equação modificada de Karadinos (1976) se aproximaram de zero (Tabela 2), sem significado prático em trabalhos de amostragem.

Tabela 2 - Tamanho da amostra (n) para estimar o número médio de ácaros Dichopelmus notus por folha e por planta em plantios comerciais de erva-mate, através da equação modificada de Karadinos (1976), em diferentes níveis de precisão (D\%). Chapecó, SC, 2006

Table 2 -Size sample (n) to estimate the means number of mites Dichopelmus notus for leaf and plant in commercial plantations of mate-tea through the equation changed of Karadinos (1976), for different levels of accuracy (D\%). Chapecó, SC, 2006

\begin{tabular}{cccccc}
\hline Unidade de & \multicolumn{5}{c}{$\mathrm{D}(\%)$} \\
\cline { 2 - 6 } amostragem & $5 \%$ & $10 \%$ & $15 \%$ & $20 \%$ & $30 \%$ \\
\hline Folha & 540 & 135 & 60 & 34 & 15 \\
Planta & 0,37 & 0,09 & 0,04 & 0,02 & 0,01 \\
\hline
\end{tabular}

R. Árvore, Viçosa-MG, v.33, n.1, p.143-150, 2009 
Tabela 3 - Estimativa do tamanho da amostra (n) para estimar o número médio de ácaros Dichopelmus notus por folha e por planta em plantios comerciais de erva-mate em Chapecó, SC, através da equação proposta por Cochran (1977), em diferentes níveis de precisão (D\%). Santa Maria, RS, 2006

Table 3 - Estimate of sample size ( $n$ ) to estimate the mean number of Dichopelmus notus mites per leaf and plant in commercial plantations of mate-tea in Chapecó, SC, through the equation proposed by Cochran (1977), for different levels of accuracy (D\%). Santa Maria, RS, 2006

\begin{tabular}{|c|c|c|c|c|c|c|c|c|c|c|}
\hline \multirow[b]{3}{*}{ Data } & \multicolumn{10}{|c|}{ Unidade de Amostragem } \\
\hline & \multicolumn{5}{|c|}{ Folha } & \multicolumn{5}{|c|}{ Planta } \\
\hline & D5 & D10 & D15 & D20 & D30 & D5 & D10 & D15 & D20 & D30 \\
\hline $27 / 01 / 04$ & 227 & 502 & 461 & 414 & 320 & 22 & 13 & 7 & 5 & 2 \\
\hline $12 / 02 / 04$ & 263 & 512 & 481 & 443 & 361 & 24 & 15 & 9 & 6 & 3 \\
\hline $28 / 02 / 04$ & 476 & 536 & 531 & 524 & 505 & 29 & 26 & 23 & 19 & 13 \\
\hline $17 / 03 / 04$ & 532 & 540 & 539 & 539 & 537 & 30 & 30 & 29 & 29 & 27 \\
\hline $02 / 04 / 04$ & 537 & 540 & 540 & 539 & 538 & 30 & 30 & 30 & 29 & 28 \\
\hline $16 / 04 / 04$ & 529 & 540 & 539 & 539 & 537 & 30 & 30 & 29 & 29 & 27 \\
\hline $03 / 05 / 04$ & 501 & 537 & 534 & 530 & 518 & 29 & 28 & 25 & 22 & 17 \\
\hline $18 / 05 / 04$ & 387 & 521 & 499 & 470 & 405 & 26 & 18 & 12 & 8 & 4 \\
\hline 04/06/04 & 263 & 513 & 482 & 446 & 366 & 24 & 15 & 10 & 6 & 3 \\
\hline $17 / 06 / 04$ & 284 & 505 & 466 & 422 & 331 & 23 & 13 & 8 & 5 & 2 \\
\hline $01 / 07 / 04$ & 269 & 501 & 460 & 413 & 319 & 22 & 13 & 7 & 5 & 2 \\
\hline $17 / 07 / 04$ & 201 & 479 & 420 & 357 & 251 & 19 & 9 & 5 & 3 & 1 \\
\hline 03/08/04 & 307 & 521 & 499 & 472 & 407 & 26 & 18 & 12 & 8 & 4 \\
\hline $19 / 08 / 04$ & 287 & 517 & 490 & 457 & 384 & 25 & 17 & 11 & 7 & 4 \\
\hline $03 / 09 / 04$ & 404 & 530 & 518 & 501 & 460 & 28 & 22 & 17 & 13 & 7 \\
\hline $17 / 09 / 04$ & 446 & 536 & 531 & 525 & 507 & 29 & 27 & 23 & 20 & 14 \\
\hline $01 / 10 / 04$ & 299 & 517 & 491 & 458 & 385 & 25 & 17 & 11 & 7 & 4 \\
\hline $15 / 10 / 04$ & 481 & 533 & 525 & 513 & 483 & 28 & 24 & 20 & 15 & 10 \\
\hline $28 / 10 / 04$ & 300 & 512 & 481 & 443 & 362 & 24 & 15 & 9 & 6 & 3 \\
\hline $11 / 11 / 04$ & 176 & 495 & 449 & 396 & 297 & 21 & 11 & 6 & 4 & 2 \\
\hline $26 / 11 / 04$ & 75 & 336 & 228 & 157 & 83 & 8 & 3 & 1 & 1 & 0 \\
\hline $09 / 12 / 04$ & 301 & 526 & 510 & 490 & 438 & 27 & 21 & 15 & 11 & 6 \\
\hline $22 / 12 / 04$ & 327 & 524 & 505 & 481 & 424 & 26 & 19 & 13 & 9 & 5 \\
\hline $10 / 01 / 05$ & 436 & 533 & 524 & 512 & 482 & 28 & 24 & 19 & 15 & 9 \\
\hline
\end{tabular}

A variabilidade nos níveis de infestação do ácaro entre datas, tendo a planta como unidade de amostragem, pode ter levado a esse resultado, ou seja, valores do coeficiente "a" próximos a zero. O valor do coeficiente "b" $(\hat{b}=1,8047)$ acusa maior sensibilidade da variância em relação à infestação média quando se trabalha com a planta do que com amostras de folhas $(\hat{b}=1,7519)$. O valor mais elevado de "b", para plantas, evidenciou tendência de maior agregação entre estas do que entre folhas. Isso também pode ser visualizado pela grande diferença entre a menor $(10,09)$ e a maior variância $(15.564,67)$ nas plantas (Figura 2B). A discrepância na amostragem das folhas foi elevada (0,25 e 306,08), mas, proporcionalmente, menor (Figura $2 \mathrm{~A}$ ).

$\mathrm{O}$ teste de Bartlett detectou heterogeneidade de variâncias do número de ácaros entre folhas $\left(\chi^{2}=20.310,89\right.$, $p$-valor $<0,0001)$ e plantas $\left(\chi^{2}=1.122,50, p\right.$-valor $<$ $0,0001)$, como valores altamente significativos.
Verifica-se grande variação no número de amostras em decorrência da época de avaliação e nível de precisão considerado (Tabela 3 ). Por exemplo, para D $=15 \%$ o número de folhas a serem avaliadas varia de $228 \mathrm{em}$ uma única planta a 540 folhas coletadas em 30 plantas. No entanto, as amostras mantiveram-se mais estáveis no período de maior infestação, fevereiro a maio, variando entre 481 e 540 folhas. Mesmo assim, é difícil chegar a uma recomendação tecnicamente viável, pois nos valores obtidos no dia 28/02/2004 (início do aumento da infestação do ácaro), para $\mathrm{D}=15 \%$, seria necessário amostrar 531 folhas em 23 plantas, ou seja, uma média de 23,08 folhas por planta. Com isso, o processo de amostragem seria muito dispendioso com os trabalhos de monitoramento dos níveis de infestação do ácaro realizados em intervalos quinzenais, uma vez que seu ciclo biológico se completa em aproximadamente 10 dias. Além disso, há várias outras atividades que necessitam de atenção num programa de manejo 
integrado da cultura, como adubação, capinas, irrigação e podas, entre outras.

Diante dos resultados, uma das possibilidades para obter uma estimativa do número médio de ácaros por folha em cultivos comerciais de erva-mate, com nível de precisão de $15 \%$, é amostrar 60 folhas em intervalos quinzenais (determinada pela equação modificada de Karadinos (1976) em 22 plantas (média das amostras do período de fevereiro a maio, calculada pelo método apresentado por Cochran (1977)).

Pode-se amostrar cerca de três folhas por planta de erva-mate ao acaso numa área de aproximadamente $7.500 \mathrm{~m}^{2}$. Caso o talhão seja de 1 ha, pode-se trabalhar com 29 plantas, sendo a amostragem realizada, preferencialmente, de fevereiro a abril, período de maior infestação na região onde foi realizado o estudo. Esse procedimento garante a praticidade desejada em trabalhos de monitoramento e mantém boa precisão na obtenção das estimativas amostrais.

\section{CONCLUSÕES}

A infestação do ácaro-do-bronzeado Dichopelmus notus Keifer (Acari: Eriophyidae) ocorre de forma agregada nos plantios comerciais de erva-mate do oeste catarinense, tanto na folha quanto na planta.

Três folhas de erva-mate devem ser amostradas em cada 22 plantas, ao acaso, em talhões com $7.500 \mathrm{~m}^{2}$, ou em 29 plantas por hectare, de fevereiro a abril, para obter uma estimativa do número de ácaros por folha.

\section{AGRADECIMENTOS}

Ao CNPq, à CAPES e à FAPERGS, pelos recursos financeiros para a realização deste trabalho e pelas bolsas de produtividade em pesquisa.

\section{REFERÊNCIAS}

BARBOSA, J. C. Métodos estatísticos aplicados à entomologia. Jaboticabal: DCE/ FCAV/UNESP, 2001. 250p.

BEARZOTI, E.; AQUINO, L. H. Plano de amostragem seqüencial para avaliação de infestação de bicho-mineiro (Lepidoptera: Lyonetiidae) no sul de Minas Gerais.

Pesquisa Agropecuária Brasileira, v.29, n.5, p.695-705, 1994.
COCHRAN, W. G. Sampling techniques. 3.ed. Ney York: John Wiley, 1977.

FARIAS, P. R. S. et al. Amostragem seqüencial com base na lei de Taylor para levantamento de Spodoptera frugiperda na cultura do milho. Scientia Agrícola, v.58, n.2, p.395-399, 2001.

GRECO, N. M. et al. Spatial distribution and coincidence of Neoseiulus californicus and Tetranychus urticae (Acari: Phytoseiidae, Tetranychidae) on strawberry. Experimental and Applied Acarology, v.23, n.7, p.567-579, 1999.

IWAO, S. Application of the m-m method to the anaysis of spatial patterns by changing the quadrat zise. Research on Population Ecology, v.14, p.97-128, 1972.

KARADINOS, M. G. Optimum sampling size and comments on some publisched formulae.

\section{Bulletin Entomology Society} American, v.22, p.417-421, 1976.

LLOYD, M. Mean crowding. Journal of Animal Ecology, v.36, n.1, p.1-30, 1967.

MARUYAMA, W. I. et al. Distribuição Espacial de Dilobopterus costalimai Young (Hemiptera:

Cicadellidae) em Citros na Região de Taquaritinga, SP. Neotropical Entomology, v.31, n.1, p.35-40, 2002.

ONZO, A. et al. Temporal and spatial dynamics of an exotic predatory mite and its herbivorous mite prey on cassava in Benin, West Africa. Environmental Entomology, v.34, n.4, p.866-874, 2005.

PENTEADO, S. R. C. Principais pragas da ervamate e medidas para o seu controle. In: Ervamate: biologia e cultura no Cone Sul. Porto Alegre: Universidade Federal do Rio Grande do Sul, 1995. 356p.

PEREIRA, M. F. A. et al. Amostragem seqüencial (presença-ausência) para Bemisia tabaci (Genn.) biótipo B (Hemiptera: Aleyrodidae) em feijoeiro (Phaseolus vulgaris L.). Neotropical

Entomology, v.33, n.4, p.499-504, 2004.

REYNOLDS, E.B. et al. Size shape and replications of plots for field, experiments with cotton.

Agronomy Journal, v.26, n.9, p.725-734, 1934. 
RIBEIRO, L. G.; VILLACORTA, A.; FOERSTER, L. A. Plano de amostragem de presença-ausência para Panonychus ulmi (Koch, 1836) (Acari: Tetranychidade) em macieira. Anais da Sociedade Entomológica do Brasil, v.19, n.1, p.221-230, 1990.

RUESINK, W. G.; KOGAN, M. The quantitative basis of pest management: sampling and measuring. In: METCALF, R. L.; MUCKMANN, W. H. Introduction to insect pest management. New York: John Willey, 1980. p.315-352.

SANTANA, D. L. et al. Ácaros em erva-mate. In.: CONGRESSO SUL-AMERICANO DE ERVA-MATE, 1., 1997, Curitiba Anais... Curitiba: Embrapa-CNPF, 1997. p.464. (Documento, 33).

SILVA, L. et al. Sampling plans for Pseudaletia unipuncta (Lepidoptera: Noctuidae) larvae in azorean pastures. Environmental

Entomology, v.32, n.5, p.1211-1218, 2003.

SILVEIRA NETO, S. et al. Manual de ecologia de insetos. São Paulo: Agronomica Ceres, 1976. 419p.
STEEL, R. G. D. et al. Principles and procedures of statistics: a biometrical approach. 3.ed. New York: McGraw Hill Book, 1997. 666p.

TAYLOR, L. R. Agregation, variance and the mean. Nature, v.189, n.4766, p.732-735, 1961.

VIEIRA NETO, J.; CHIARADIA, L. A. Plano de amostragem para Dichopelmus notus Keifer (Acari, Eriophyidae). Pesquisa Agropecuária Gaúcha, v.5, n.3, p.357-361, 1999.

WILSON, L. T. et al. Presence-absence sampling for cabbage aphid and green peach aphid (Homoptera: Aphididae) on Brussels sprouts.

Journal of Economic Entomology, v.76, n.3, p.476-479, 1983.

WILSON, L. T. et al. Sampling mites in almonds: I. Within-tree distribution and clumping pattern of mites with comments on predator-prey interactions. Hilgardia, v.52, n.1, p.1-13, 1984.

WILSON, L. T.; ROOM, P. M. Clumping patterns of fruit and arthropods in cotton with implications for binomial sampling. Environmental Entomology, v.12, n.1, p.50-54, 1983. 